Pacific Journal of Mathematics

THREE THEOREMS ON IMBEDDED PRIME DIVISORS O 


\title{
THREE THEOREMS ON IMBEDDED PRIME DIVISORS OF PRINCIPAL IDEALS
}

\author{
L. J. RATLIFF, JR.
}

Let $B$ be a finitely generated integral domain over a Noetherian domain $A$. The first theorem shows that there are only finitely many imbedded prime divisors of principal ideals in $B$ if and only if this holds in $A$. The second theorem gives a necessary and sufficient condition in order that only finitely many height one prime ideals in $A$ ramify in $B$, when $A$ is locally factorial. The third theorem characterizes local domains which contain infinitely many imbedded prime divisors of principal ideals.

1. Introduction. For convenience of description, let $\mathscr{F}(C)$ denote the set of imbedded prime divisors of principal ideals generated by regular elements in a ring $C$. Then a desirable property of a Noetherian domain $A$ is for $\mathscr{J}(A)$ to be a finite set, for this implies that there exist nonzero elements $a \in A$ such that no principal ideal in $A_{a}$ has an imbedded prime divisor, hence $A_{a}=A_{a}^{(1)}$, and so $A_{a}$ has certain other nice properties. Theorem 2.4 shows that this desirable property is inherited by finitely generated extension domains of $A$, and its Corollary (2.8) extends this result to the case $A$ contains nonzero zero divisors. Corollary 2.9 shows that for a large class of Noetherian rings $A, \mathcal{F}(B)$ is a finite set (where $B$ is a certain type of finitely generated extension ring of $A$ ).

In $\S 3$, (3.1) gives two characterizations of a local domain $R$ such that $\mathscr{F}(R)$ is an infinite set. That $\mathscr{F}(R)$ can be an infinite set is closely related to some open problems on unmixed and quasi-unmixed local domains, and characterizations of such $R$ are therefore important. Corollary 3.4 extends these characterizations to certain local rings, and then an example is given of a local domain $L$ such that: $L^{(1)}$ is not a finite $L$-algebra; $\mathscr{F}(L)$ is a finite set; and, $\mathscr{F}\left(L^{*}\right)$ is an infinite set, where $L^{*}$ is the completion of $L$. This example also gives some information concerning the open problem of whether an integrally closed local domain must be unmixed.

Section 4 is concerned with unramification of height one prime ideals. Such unramification is of importance in a numbe of problems in local ring theory, for example in the purity of the branch locus [5, (41.1)]. Theorem 4.4 gives two necessary and sufficient conditions in order that only finitely many height one prime ideals in a locally factorial Noetherian domain $A$ ramify in a finite separably generated extension domain $B$ of $A$. The paper is closed with some corollaries 
to (4.4), among these are that the conditions are satisfied if either $A$ is a Dedekind domain (4.6) or $B$ is a flat $A$-algebra (4.8).

2. Finite extension rings. All rings in this paper are assumed to be commutative rings with a unit, and the undefined terminology in the paper is, in general, the same as that in [5].

We begin with the following definition.

Definition 2.1. For a ring $A$, let $\mathscr{J}(A)=\{p \in \operatorname{Spec} A ; p$ is an imbedded prime divisor of a principal ideal generated by a regular element in $A$ \}.

That $\mathscr{J}(A)$ must be infinite in many cases when $A$ contains nonzero zero divisors follows from the following remark. It is because of this remark that we shall usually only consider rings with no imbedded prime divisors of zero in this paper.

REMARK 2.2. (Cf. [6, Lemma 6].) Let $q$ be a prime divisor of zero in a Noetherian ring $A$, and let $b$ be a regular element in $A$ such that $(q, b) A \neq A$. Then each minimal prime divisor of $(q, b) A$ is a prime divisor of $b A$.

Proof. This follows immediately from [6, Lemma 6] and its proof.

The following lemma sets the stage for an easy proof of the first theorem in this paper, and provides information needed throughout the paper. Although the lemma could be adapted to the case $A$ contains nonzero zero divisors, it is stated only for the integral domain case, since this case is sufficient for our purposes in this paper. The following known fact is needed for the proof of (2.3): If $B$ is a Noetherian ring which is a flat extension of a Noetherian ring $A$ and $I$ is an ideal in $A$, then a prime ideal $P$ in $B$ is a prime divisor (resp., minimal prime divisor) of $I B$ if and only if $P$ is a prime divisor (resp., minimal prime divisor) of $p B$, for some prime divisor (resp., minimal prime divisor) $p$ of $I$, in which case $P \cap A=p[5,(18.11)]$. Also, implicitly used in the proof (and frequently throughout this paper) is the fact that if $p \in \mathscr{J}(A)$ and $a$ is a regular element in $p$, then $p$ is an imbedded prime divisor of $a A$ [5, (12.6)] (so the intersection of infinitely many such $p$ consists of zero divisors).

Lemma 2.3. Let $A$ be a Noetherian domain.

(1) For each nonzero $b$ in $A, \mathscr{I}\left(A_{b}\right)=\left\{p A_{b} ; b \notin p \in \mathscr{J}(A)\right\}$, and $\mathscr{I}(A)=\left\{P \cap A ; P \in \mathscr{S}\left(A_{b}\right)\right\} \cup\{p ; p$ is an imbedded prime divisor of $b A\}$. 
(2) For each $n \geqq 1, \mathscr{J}\left(A_{n}\right)=\left\{p A_{n} ; p \in \mathscr{J}(A)\right\}$, where $A_{n}=$ $A\left[X_{1}, \cdots, X_{n}\right]$ with the $X_{i}$ indeterminates.

(3) If $A^{\prime}$ is a finite free integral extension ring of $A$, then $\mathscr{J}\left(A^{\prime}\right)=\left\{p^{\prime} \in \operatorname{Spec} A^{\prime} ; p^{\prime} \cap A \in \mathscr{I}(A)\right\}$.

(4) If $A^{*}$ is a flat A-algebra, then $\mathscr{J}\left(A^{*}\right) \supseteq\left\{p^{*} \in \operatorname{Spec} A^{*} ; p^{*}\right.$ is a prime divisor of $p A^{*}$, for some $\left.p \in \mathscr{J}(A)\right\}$.

(5) $\mathscr{S}(A)$ is a finite set if and only if $A^{(1)}=\bigcap\left\{A_{p} ; p \in \operatorname{Spec} A\right.$ and height $p=1\} \subseteq A_{b}$, for some nonzero $b$ in $A$.

(6) If $B$ is a finitely generated algebraic extension domain of $A$, then $\mathscr{I}(B)$ is a finite set if and only if $\mathscr{J}(A)$ is a finite set.

Proof. (1) and (2) are straightforward, (3) and (4) follow from [5, (18.11)], and (5) is given in [7, Lemma 5.15(8)] (see (3.3) below). It clearly suffices to prove (6) in the case $B=A[b]$, for some $b \in B$. Then there exists a nonzero $a \in A$ such that $A_{a}[b]=B_{a}$ is a free integral extension domain of $A_{a}$. Therefore, it follows from (1) and (3) that $\mathscr{J}\left(B_{a}\right)$ is a finite set if and only if $\mathscr{J}(A)$ is, hence, by (1), $\mathscr{J}(B)$ is a finite set if and only if $\mathscr{J}(A)$ is a finite set.

THEOREM 2.4. Let $B$ be a finitely generated integral domain over a Noetherian domain $A$. Then $\mathcal{J}(B)$ is a finite set if and only if $\mathscr{S}(A)$ is a finite set.

Proof. There are elements $X_{1}, \cdots, X_{n}$ in $B$ which are algebraically independent over $A$ such that $B$ is a (finite) algebraic extension domain of $A_{n}=A\left[X_{1}, \cdots, X_{n}\right]$. Therefore, the conclusion follows from (2.3)(2) and (6).

To generalize (2.4) to the case where $A$ contains nonzero divisors of zero, the following two lemmas are needed.

Lemma 2.5. Let $q$ be a minimal prime ideal in a Noetherian ring $A$. Then there exists $a \in A, \notin q$ such that, for all $P \in \operatorname{Spec} A$ such that $q \subseteq P$ and $a \notin P, P \in \mathscr{J}(A)$ if and only if $P / q \in \mathscr{J}(A / q)$.

Proof. If $b \in A, \notin q$, then it clearly suffices to prove the lemma for $A_{b}$ instead of $A$. Hence it may be assumed that $q$ is nilpotent. The lemma now readily follows from [3, IV. (6.10.6)]. Specifically, the referenced result is essentially local, and passing from the language of preschemes to the language of commutative rings we find that it asserts that there exists $a \in A, \notin q$ such that, for all $P \in \operatorname{Spec} A$ such that $q \leqq P$ and $a \notin P$, altitude $A_{P}=$ altitude $(A / q)_{P / q}$ and Prof $A_{P}=$ Prof $(A / q)_{P / q}$ (since $q$ is nilpotent), where Prof $R$ is the length of a maximal $R$-sequence with $R$ a local ring. The lemma follows from this, since $P \in \mathscr{J}(A)$ if and only if Prof $A_{P}=1$ and height $P>1$. 
Lemma 2.6. Let $A$ be a Noetherian ring such that each prime divisor of zero in $A$ is minimal. Then $\mathscr{J}(A)$ is a finite set if and only if, for each minimal prime ideal $q$ in $A, \mathcal{F}(A / q)$ is a finite set.

Proof. Let $q_{1}, \cdots, q_{g}$ be the minimal prime ideals in $A$, and, for $i=1, \cdots, g$, let $a_{i}$ be as in (2.5) for $q_{i}$. Therefore, for all $P \in \operatorname{Spec} A$ such that $q_{i} \subseteq P$ and $a_{i} \notin P, P \in \mathscr{J}(A)$ if and only if $P / q_{i} \in \mathscr{J}\left(A / q_{i}\right)$. Now, if $\mathscr{J}(A)$ is an infinite set, then there is an $i$ such that $q_{i}=$ $\bigcap\left\{P ; q_{i} \subset P \in \mathscr{I}(A)\right\}$. Fix such an $i$, and let $q=q_{i}$ and $a=a_{i}$. Then $q A_{a}=\bigcap\left\{P A_{a} ; q A_{a} \subset P A_{a} \in \mathscr{J}\left(A_{a}\right)\right\}$. Hence (2.5) implies $\mathscr{J}(A / q)$ is an infinite set, since $\mathscr{J}\left((A / q)_{a+q}\right)$ is. Conversely, if $\mathscr{J}\left(A / q_{i}\right)$ is an infinite set, for some $i=1, \cdots, g$, then fix such an $i$ and let $q=q_{i}$ and $a=a_{i}$. Then $(0)=\bigcap\{P / q, P / q \in \mathscr{J}(A / q)\}$, so $(0)(A / q)_{a+q}=$ $\bigcap\left\{(P / q)_{a+q} ;(P / q)_{a+q} \in \mathscr{J}\left((A / q)_{a+q}\right)\right\}$, hence $(2.5)$ implies $\mathscr{J}(A)$ is an infinite set.

Corollary 2.7. With $A$ as in (2.6), $\mathscr{I}(A)$ is a finite set if and only if $\mathscr{S}(A /(\operatorname{Rad} A))$ is a finite set.

\section{Proof. Clear by (2.6).}

COROLlaRY 2.8. Let $B$ be a finitely generated ring over a Noetherian ring $A$, and assume that all prime divisors of zero in $A$ and in $B$ are minimal and that, for each prime divisor $q$ of zero in $B, q \cap A$ is a prime divisor of zero. Then $\mathscr{S}(A)$ is a finite set if and only if $\mathscr{\rho}(B)$ is a finite set.

Proof. Let $q_{1}, \cdots, q_{g}$ be the prime divisors of zero in $B$. Then, by (2.6) and $(2.4), \mathscr{I}(B)$ is a finite set if and only if $\mathscr{I}\left(B / q_{i}\right)$ is, for all $i$, if and only if $\mathscr{J}\left(A /\left(q_{i} \cap A\right)\right)$ is, for all $i$, if and only if $\mathscr{J}(A)$ is a finite set.

The corollary shows that such finite extension rings of a large class of Noetherian rings have only finitely many imbedded prime divisors of principal ideals. Specifically, the following corollary holds.

Corollary 2.9. Let $A$ and $B$ be as in (2.8). Then $\mathscr{J}(B)$ is finite in each of the following cases:

(1) $A$ is locally factorial (4.1).

(2) $A$ is integrally closed.

(3) $A$ is pseudogeometric [5, p. 131].

(4) $A$ is Japanese [2, 0. (23.1.1)].

(5) The integral closure of $A$ is a finite A-algebra.

(6) $A$ is locally Macaulay. 
(7) $A$ is excellent [3, IV, (7.8.2)].

(8) Altitude $A \leqq 1$.

(9) $A$ is semi-local and altitude $A \leqq 2$.

(10) $A$ is an analytically unramified semi-local ring.

(11) $A$ is an unmixed semi-local domain.

(12) $A$ is a local ring whose completion has no imbedded prime divisor of zero.

Proof. By (2.8) it may be assumed, where appropriate, that $\operatorname{Rad} A=(0)$. Then it is well known that in each case $\mathscr{J}(A)$ is a finite set (for (11), see (3.5)(2) below and (2.3)(5), and for (12), see (3.4) below), hence the conclusion follows from (2.8).

One further result which is related to (2.4) and which gives a sharper conclusion when $\mathscr{J}(A)$ is finite is given in the following proposition.

Proposition 2.10. Let $A$ and $B$ be as in (2.4), and assume $\mathscr{F}(A)$ is a finite set. Then there exists a nonzero $a$ in $A$ such that $B_{a}^{(1)}$ is a finite $B_{a}$-algebra.

Proof. It is known [5, (14.4)] that there exist $X_{1}, \cdots, X_{n}$ in $B$ which are algebraically independent over $A$ and a nonzero $c$ in $A$ such that $B_{c}$ is a (finite) integral extension domain of $A\left[1 / c, X_{1}, \cdots, X_{n}\right]$. Also, since $\mathscr{J}(A)$ is a finite set, there is a nonzero $d$ in $A$ such that $A^{(1)} \cong A_{d}(2.3)(5)$. Then $A_{d}^{(1)}=A_{d}$ [7, Corollary 5.9(2)], so, with $a=$ $c d, A_{a}^{(1)}=A_{a}\left[7\right.$, Corollary 5.9(2)], hence $D^{(1)}=D$ [7, Lemma 5.11(2)], where $D=A\left[1 / a, X_{1}, \cdots, X_{n}\right]$. Therefore, since $B_{a}$ is a finite integral extension demain of $D, B_{a}^{(1)}$ is a finite $B_{a}$-algebra [3, IV. (5.10.17)].

3. The local domain case. With $A$ and $B$ as in (2.8), if $A$ is semi-local of altitude at most two, then $\mathscr{J}(B)$ is a finite set (2.9)(9). This leads to the question: Can $\mathscr{J}(R)$ be an infinite set when $R$ is a local ring? The answer is well known to be "yes", if there is an imbedded prime divisor $q$ of zero in $R$ such that depth $q>1$ (2.2). Quite recently the answer was shown to be "yes" even when $R$ is a local domain. Specifically, in [1, Proposition 3.5] an example was constructed of a local domain $R$ such that altitude $R=3$ and $R$ contains infinitely many height two prime ideals $P$ such that $R_{P}$ is not Macaulay (hence $P R_{P}$, and so $P$ also, is an imbedded prime divisor of each nonzero principal ideal that is contained in it).

The following theorem gives two characterizations of a local domain $R$ such that $\mathscr{J}(R)$ is an infinite set.

THEOREM 3.1. The following statements are equivalent for a 
local domain $(R, M)$ :

(1) $\mathscr{F}(R)$ is an infinite set.

(2) For no nonzero $b \in R$ is $R^{(1)} \cong R_{b}$.

(3) There exists an imbedded prime divisor $q$ of zero in the $M$ adic completion $R^{*}$ of $R$ such that $q=\bigcap\left\{p^{*} \in \mathscr{F}\left(R^{*}\right) ; q \subset p^{*}\right.$ and $\left.p^{*} \cap R \in \mathscr{I}(R)\right\}$.

Proof. The equivalence of (1) and (2) has already been noted in (2.3)(5). For the equivalence of (1) and (3), assume first that (1) holds. For each $p \in \mathscr{F}(R)$, let $p^{*}$ be a minimal prime divisor of $p R^{*}$, and let $\mathscr{J}^{*}=\left\{p^{*} ; p \in \mathscr{J}(R)\right\}$ (so $\mathscr{J}^{*} \subseteq \mathscr{J}\left(R^{*}\right)(2.3)(4)$ ). Then, for each $p^{*} \in \mathscr{I}^{*}, R_{p^{*}}^{*}$ is not Macaulay, so $I \subseteq p^{*}$, where $I$ is the radical ideal in $R^{*}$ which defines the non-Macaulay locus of $R^{*}$ (the Macaulay locus of $R^{*}$ (that is, the set $\left\{p^{*} \in \operatorname{Spec} R^{*} ; R_{p^{*}}^{*}\right.$ is Macaulay\}) is open (in the Zariski topology on Spec $R^{*}$ ) [3, IV. (5.11.8)]). Now infinitely many $p^{*}$ must contain some (minimal) prime divisor of $I$, say $q$, and then $q \subseteq \bigcap\left\{p^{*} ; q \subseteq p^{*} \in \mathscr{J}^{*}\right\}$. Since $R^{*}$ is Noetherian, it follows that $q=$ $\bigcap\left\{p^{*} ; q \subset p^{*} \in \mathscr{J}^{*}\right\}$ and $q$ is a prime divisor of zero. Since $R_{q}^{*}$ is not Macaulay, height $q>0$, so (3) holds.

Conversely, if (3) holds, then let $\mathscr{J}=\left\{p^{*} \cap R ; q \subset p^{*} \in \mathscr{F}\left(R^{*}\right)\right.$ and $\left.p^{*} \cap R \in \mathscr{J}(R)\right\}$, so $\mathscr{J} \subseteq \mathscr{J}(R)$ and (1) holds if $\mathscr{J}$ is an infinite set. Since $q$ is a prime divisor of zero, there are infinitely many $p^{*}$, so (1) holds if, for each $p^{*} \cap R \in \mathscr{F}, p^{*}$ is a prime divisor of $\left(p^{*} \cap R\right) R^{*}$. But this is true by [5, (18.11)], since $p^{*} \in \mathscr{J}\left(R^{*}\right)$ and $p^{*} \cap R \in \mathscr{J}(R)$.

REMarks 3.2. (1) Possibly the theorem remains true if the set in (3) is replaced by $\left\{p^{*} \in \operatorname{Spec} R^{*} ; q \subset p^{*}\right.$ and $\left.p^{*} \cap R \in \mathscr{F}(R)\right\}$. At least the author knows of no case where this last set does not work. Of course, this last set always works in the case that infinitely many of the $p^{*}$ satisfy: height $p^{*}=$ height $q+1$ (2.2).

(2) The proof that (1) $\Rightarrow(3)$ shows that, if $\mathscr{J}\left(R^{*}\right)$ is an infinite set, then $R^{*}$ has an imbedded prime divisor of zero. The converse is not true, as is seen if altitude $R=2$.

(3) If altitude $R=2$ and $R^{*}$ has an imbedded prime divisor of zero, then $R^{(1)}$ is not a finite $R$-algebra (3.5)(1) but $R^{(1)} \cong R_{b}$, for each nonzero $b \in M$. For an example of this, see [1, Proposition 3.3].

(4) It is easy to see that if altitude $R=3$, then $\mathscr{J}(R)$ is an infinite set if and only if the Macaulay locus of $R$ is not open. For, the Macaulay locus of $R$ is open if and only if $\mathscr{J}(R)$ is a finite set, when altitude $R=3$.

To generalize (3.1) to the case where $R$ contains nonzero zero divisors, the following lemma is needed.

Lemma 3.3. Let $A$ be a Noetherian ring which has no imbedded 
prime divisors of zero, and let altitude $A>0$. Then $\mathscr{J}(A)$ is a finite set if and only if $A^{(1)}=\bigcap\left\{A_{(p)} ; p \in \operatorname{Spec} A\right.$, height $p=1$, and $p$ contains a regular element $\} \subseteq A_{b}$, for some regular element $b \in A$, where $A_{(p)}=\{a / c ; a \in A$ and $c$ is a regular element in $A$ and not in $p\}$.

Proof. If $\mathscr{J}(A)$ is a finite set, then, since altitude $A>0$, let $b$ be a regular element in $\bigcap\{P ; P \in \mathscr{J}(A)\}$, so $\mathscr{F}\left(A_{b}\right)$ is empty. Hence $A_{b}=$ [8, Corollary 2.18(2)] $A_{b}^{(1)}=\left(A^{(1)}\right)_{b} \supseteqq A^{(1)}$. Conversely, if $A^{(1)} \subseteq A_{b}$, then $\left(A^{(1)}\right)_{b}=A_{b} \subseteq A_{b}^{(1)}=\left(A^{(1)}\right)_{b}$, so $A_{b}=A_{b}^{(1)}$, hence $b \in \bigcap\{P ; P \in \mathscr{J}(A)\}$, by [8, Corollary 2.18(2)]. Thus $\mathscr{J}(A)$ is a finite set, since $b$ is regular and $b A$ has only finitely many prime divisors.

COROLLARY 3.4. Let $R$ be a local ring which has no imbedded prime divisors of zero, let altitude $R>0$, and let $R^{*}$ be the completion of $R$. Then the following statements are equivalent:

(1) $\mathscr{S}(R)$ is an infinite set.

(2) For no regular element $b \in R$ is $R^{(1)} \subseteq R_{b}$.

(3) There exists an imbedded prime divisor $q^{*}$ of zero in $R^{*}$ such that $q^{*}=\bigcap\left\{P \in \mathscr{J}\left(R^{*}\right) ; q^{*} \subset P\right.$ and $\left.P \cap R \in \mathscr{J}(R)\right\}$.

Proof. (1) $\Leftrightarrow(2)$ is given by (3.3).

$\mathscr{J}(R)$ is an infinite set if and only if $\mathscr{J}(R / q)$ is, for some (minimal) prime divisor $q$ of zero (2.6), and $\mathscr{J}(R / q)$ is an infinite set if and only if there is an imbedded prime divisor $q^{* \prime}=q^{*} / q R^{*}$ of zero in $R^{*} / q R^{*}$ (equivalently, there exists an imbedded prime divisor $q^{*}$ of zero in $R^{*}$ [5, (18.11)]) such that $q^{* \prime}=\bigcap\left\{P^{\prime} \in \mathscr{F}\left(R^{*} / q R^{*}\right) ; q^{* \prime} \subset P^{\prime}\right.$ and $\left.P^{\prime} \cap(R / q) \in \mathscr{J}(R / q)\right\}$ (3.1). By (2.5), there exists $a \in R, \notin q$ such that, for all $Q \in \operatorname{Spec} R$ such that $q \subseteq Q$ and $a \notin Q, Q \in \mathscr{J}(R)$ if and only if $Q / q \in \mathscr{J}(R / q)$. Since $a+q$ is a regular element in $R / q, q^{* \prime}=$ $\bigcap\left\{P^{\prime} \in \mathscr{J}\left(R^{*} / q R^{*}\right) ; q^{* \prime} \subset P^{\prime}, P^{\prime} \cap(R / q) \in \mathscr{J}(R / q)\right.$, and $\left.a+q \notin P^{\prime} \cap(R / q)\right\}$; so, by (2.5), $\mathscr{J}(R)$ is an infinite set if and only if there is an imbedded prime divisor $q^{*}$ of zero in $R^{*}$ such that $q^{*}=\bigcap\left\{P ; P^{\prime}=P / q R^{*} \epsilon\right.$ $\mathscr{I}\left(R^{*} / q R^{*}\right), q^{*} \subset P$, and $\left.P \cap R \in \mathscr{F}(R)\right\}$. Since $P^{\prime}$ is a prime divisor of $\left(P^{\prime} \cap(R / q)\right)\left(R^{*} / q R^{*}\right)=((P \cap R) / q)\left(R^{*} / q R^{*}\right)=\left((P \cap R) R^{*}\right) / q R^{*}$ (since $P^{\prime} \in \mathscr{J}\left(R^{*} / q R^{*}\right)$ and $\left.P^{\prime} \cap(R / q) \in \mathscr{J}(R / q)\right), P$ is a prime divisor of $(P \cap R) R^{*}$, hence $P \in \mathscr{J}\left(R^{*}\right)(2.3)(4)$.

Example 3.6 below shows somewhat more than (3.2)(3). For the example the following information is needed.

REMARKS 3.5. Let $(R, M)$ be a local domain.

(1) [3, IV. (7.2.3)]. $R^{(1)}$ is a finite $R$-algebra if and only if the following condition holds: If $q \subset p$ are prime ideals in the $M$-adic 
completion $R^{*}$ of $R$ such that $q$ is a prime divisor of zero and height $p \cap R \geqq 2$, then height $p / q \geqq 2$.

(2) [7, Lemma 5.11(1)]. If $R$ is unmixed, then $R^{(1)}$ is a finite $R$-algebra.

If $R^{(1)}$ is a finite $R$-algebra, then clearly $R^{(1)} \subseteq R_{b}$, for some nonzero $b$ in $R$. On the other hand, if $R^{(1)}$ is not a finite $R$-algebra, then there is at least one prime ideal $p$ in $R^{*}$ such that $p$ contains a prime divisor $q$ of zero such that height $p / q=1<$ height $p \cap R$ (3.5) (1), and necessarily $p \cap R \in \mathscr{I}(R)$ (since $p \in \mathscr{I}\left(R^{*}\right)$ (2.2), so $p$ is a prime divisor of $b R^{*}$, for each nonzero $b \in p \cap R$, hence $p \cap R$ is a prime divisor of each such $b R[5,(18.11)])$. Since there is one such $p$, it might be asked if there are necessarily infinitely many such if depth $q>1$ (so $p \neq M^{*}$ ). The answer is "no". In fact, the example below shows the stronger result that $\mathscr{F}(R)$ can be finite in this case.

ExAmple 3.6. A local domain $L$ such that $L^{(1)}$ is not a finite $L$ algebra and each imbedded prime divisor of zero in the completion of $L$ has depth greater than one, but $L^{(1)} \subseteq L_{b}$, for some nonzero $b$ in $L$ (hence $\mathscr{F}(L)$ is a finite set (2.3)(5)). Further, $\mathscr{I}\left(L^{*}\right)$ is an infinite set. Let $(R, M)$ be a local domain whose completion $\left(R^{*}, M^{*}\right)$ has an imbedded prime divisor of zero, and assume $\mathscr{J}(R)$ is a finite set. (For example, let $R=A$ in [1, Proposition 3.3].) Let $Q$ be an $M$ primary ideal, let $\mathscr{R}=\mathscr{R}(R, Q)=R[t Q, u]$ be the Rees ring of $R$ with respect to $Q$ ( $t$ is an indeterminate, $u=1 / t$, and $t Q=\{t m ; m \in Q\})$, and let $\mathscr{R}^{*}=\mathscr{R}\left(R^{*}, Q^{*}\right)$, where $Q^{*}=Q R^{*}$. Let $\mathscr{C}=(t Q, M, u) \mathscr{R}$ and $\mathscr{C}^{*}=\left(t Q^{*}, M^{*}, u\right) \mathscr{R}^{*}$ be the maximal homogeneous ideals in $\mathscr{R}$ and $\mathscr{R}^{*}$, respectively. Then $L=\mathscr{R}_{\mathscr{N}}$ is a dense subspace of $L^{\prime}=$ $\mathscr{R}_{\mathbb{N}^{*}}^{*}\left[7\right.$, Lemma 3.2], so, by $(3.5)(1)$, to show that $L^{(1)}$ is not a finite $L$-algebra, it suffices to prove that there exist prime ideals $q^{\prime} \subset p^{\prime}$ in $L^{\prime}$ such that $q^{\prime}$ is an imbedded prime divisor of zero and height $p^{\prime} / q^{\prime}=$ $1<$ height $p^{\prime} \cap L$ (since this will then be reproduced in the completion $L^{*}$ of $L$ and $L^{\prime}$ ). Also, since the prime divisors of zero in $L^{\prime}$ are the ideals $\left(q R^{*}[t, u] \cap \mathscr{R}^{*}\right) L^{\prime}$ with $q$ a prime divisor of zero in $R^{*}$ [9, Theorem 1.5], there are no depth one prime divisors of zero in $L^{\prime}$ (since $q R^{*}[t, u] \cap \mathscr{R}^{*} \subset M^{*} R^{*}[t, u] \cap \mathscr{R}^{*} \subset \mathscr{M}^{*}$ ), so there will be none in $L^{*}$ (since, for each prime ideal $P$ in $L^{\prime}$, each prime divisor of $P L^{*}$ has depth equal to depth $P$, by [5, (36.5) and Exercise p. 135] applied to $L^{\prime} / P$ (that $L^{\prime} / P$ satisfies the second chain condition for prime ideals follows from [7, Corollary 2.9], since $R^{*} /\left(P \cap R^{*}\right)$ does [5, (34.4)])). Let $q$ be an imbedded prime divisor of zero in $R^{*}$ and let $q^{*}=q R^{*}[t, u] \cap \mathscr{R}^{*}$, so $q^{*}$ is an imbedded prime divisor of zero in $\mathscr{R}^{*}$. Let $I=\left(q^{*}, u\right) \mathscr{R}^{*}$. Then height $q+1 \leqq$ height $I=$ (say) $h<$ height $\mathscr{L}^{*}$ (since $\mathscr{R}^{*} / q^{*} \cong \mathscr{R}\left(R^{*} / q,\left(Q^{*}+q\right) / q\right)$ [9, Theorem 2.1] and depth $q \geqq 1$, so depth $q^{*} \geqq 2$ ), and $I$ is homogeneous, so there is a 
height $h$ prime divisor $p^{*}$ of $I$ which is contained $\mathscr{C}^{*}$. Since $u \mathscr{R}^{*} \cap R^{*}=Q^{*}$ is $M^{*}$-primary, $\mathscr{R}_{p^{*} \cap \mathscr{R}}$ is a dense subspace of $\mathscr{R}_{p^{*}}^{*}$ [7, Lemma 3.2] (so height $p^{*} \cap \mathscr{R}=$ height $p^{*}=h>$ height $p^{*} / q^{*}=$ 1 ), and $p^{*} \cap \mathscr{R} \subset \mathscr{M}$. However, $\mathscr{I}(L)$ is a finite set, since $\mathscr{J}(\mathscr{R})$ is, by (2.4). Finally, $\mathscr{J}\left(L^{*}\right)$ is an infinite set, since $\mathscr{J}\left(L^{\prime}\right)$ is by (2.2) applied to $q^{*} L^{\prime}$.

REMARK 3.7. One reason for using the ring $L=\mathscr{R}_{\mathscr{M}}$ instead of $P=R[X]_{(M, X)}$ is that for the ring $L$ it is not necessary to assume that $R^{(1)}$ is not a finite $R$-algebra to prove that $L^{(1)}$ is not a finite $L$ algebra, whereas for $P$ it is necessary to assume this to prove that $P^{(1)}$ is not a finite $P$-algebra (but this does hold for $R=A$ in [1, Proposition 3.3]). A more important reason is it is an open problem if $R$ must be unmixed when $R$ is integrally closed. Related to this problem, the rings $L$ (as $Q$ varies) show that, if $R$ is integrally closed and not unmixed, then there does not exist an $M$-primary ideal $Q$ such that $L^{(1)}$ is a finite $L$-algebra.

4. Unramification of height one prime ideals. To obtain the main result of this section, the following two definitions and lemma are needed.

Definition 4.1. A ring $A$ is locally factorial in case $A_{P}$ is a unique factorization domain, for all prime ideals $P$ in $A$.

Lemma 4.2. Let $B$ be a Noetherian integral domain which contains a locally factorial Noetherian domain $A$, and let $P$ be a prime ideal in $B$ such that $P$ is a prime divisor of $p B$, for some height one prime ideal $p$ in $A$. Then the following statements hold:

(1) $P$ is a prime divisor of $q B$, for all height one prime ideals $q \subseteq P \cap A$.

(2) $P$ is an imbedded prime divisor of $p B$ if and only if $P \in$ $\mathscr{F}(B)$.

(3) $P$ is a minimal prime divisor of $p B$ if and only if height $P=1$.

Proof. Since, for each height one prime ideal $q \subseteq P \cap A, q A_{P \cap A}$ is principal and $A_{P \cap A} \subseteq B_{P}$, (1) and (2) hold, and (3) also follows from this and the Principal Ideal Theorem.

Definition 4.3. (Cf. [5, pp. 144-145].) Let $A \subset B$ be rings, let $P$ be a prime ideal in $B$, and let $p=P \cap A$. Then $P$ is unramified over $A$ in case $P B_{P}=p B_{P}$ and $B / P$ is separably generated over $A / p$ (that is, the quotient field of $B / P$ is a separable extension field of the 
quotient field of $A / p)$. If $q$ is a prime ideal in $A$, then $q B$ is $u n$ ramified over $A$ in case, for every prime divisor $Q$ of $q B, Q$ is unramified over $A$.

The following theorem is a considerable generalization of [4, Theorem 5] (see (4.6) below).

THEOREM 4.4. Let $B$ be a finite separably generated extension domain of a locally factorial Noetherian domain $A$. Then the following statements are equivalent, where $\mathscr{S}=\{p \in \operatorname{Spec} A$; height $p=1\}$ :

(1) $p B$ is unramified over $A$, for all except finitely many $p \in \mathscr{S}$.

(2) If $P$ is a height one prime ideal in $B$, then height $P \cap A \leqq$ 1 , and only finitely many of the ideals $p B$, where $p \in \mathscr{S}$, have imbedded prime divisors.

(3) If $P$ is a prime divisor of $p B$, where $p \in \mathscr{S}$, then $P \cap A=p$.

Proof. $\quad(1) \Rightarrow(2)$. By (1), with $p \in \mathscr{S}$, only finitely many $p B$ have imbedded prime divisors. Also, if $P$ is a height one prime ideal in $B$ such that height $P \cap A>1$, then clearly $P$ is a prime divisor of $p B$, for all $p \in \mathscr{S}$ such that $p \subset P \cap A$. Since there are infinitely many such $p$, (1) implies that $P$ is unramified over at least one such $p$, hence $P \cap A=p$; contradiction. Therefore, height $P \cap A \leqq 1$, as desired.

$(2) \Rightarrow(3)$. Let $p \in \mathscr{S}$ and let $P$ be a prime divisor of $p B$. If $P$ is an imbedded prime divisor of $p B$, then $P \cap A=p$, by (2) and (4.2)(1). If $P$ is a minimal prime divisor of $p B$, the height $P=1(4.2)(3)$, so (2) implies that $P \cap A=p$.

$(3) \Rightarrow(1)$. Since $B$ is a finite separably generated extension domain of $A$, there exists a separating transcendence basis $X_{1}, \cdots, X_{n}$ in $B$ of $B$ over $A$ and a nonzero element $a \in A$ such that $B_{a}$ is integrally dependent on $D=A\left[1 / a, X_{1}, \cdots, X_{n}\right][5,(39.11)]$. Then $D$ is locally factorial, since $A$ is. Let $D^{\prime}$ and $B^{\prime}$ be the integral closures of $D$ and $B$ in the quotient field of $B$, respectively, so $B_{a}^{\prime}=D^{\prime}$. Since $B_{a}$ is a finite separable algebraic extension of $D$, let $b \in B_{a}$ such that $D[b]$ and $B_{a}$ have the same quotient field, and let $d$ be the discriminant of the minimum polynomial of $b$ over the quotient field of $D$ (so $d \in D$, since $D$ is integrally closed). Then $\left.D_{d}^{\prime}=D_{d}\right)^{\prime}=D_{d}[b]$ [5, (10.17) and (10.18)], so every prime ideal in $D_{d}^{\prime}$ is unramified over $D_{d}$ [5, (38.9)]. Let $\mathcal{J}$ be the set of $p \in \mathscr{S}$ such that: (i) $a \in p$; or (ii) $d \in p D$; or (iii) $p B$ has an imbedded prime divisor. That $\mathscr{J}$ is a finite set follows from: (i) $A$ is Noetherian; (ii) $D$ is Noetherian and $p D$ is a height one prime ideal; and (iii) if $P$ is an imbedded prime divisor of $p B$, then $P \in \mathscr{J}(B)$, by (4.2)(2), and $P \cap A=p$, by (3), hence there are only finitely many such $P$ by (2.9)(1). It will now be shown that if $p \in \mathscr{S}, \notin \mathcal{J}$, then $p B$ is unramified over $A$. 
For this, note first that $p D$ is a prime ideal, $p D \cap A=p$, and $D / p D$ is a pure transcendental extension of $(A / p)_{\bar{a}}$, where $\bar{a}=a(\bmod -$ ulo $p$ ). Therefore, since every prime ideal in $D_{d}^{\prime}$ is unramified over $D_{d}, p D_{d}^{\prime}$ is unramified over $A(p \in \mathscr{S}, \notin \mathscr{J})$.

Since $p \notin \mathscr{J}, p B$ has no imbedded prime divisors, so if $P$ is a prime divisor of $p B$, then height $P=1$ (4.2)(3). Also, $a \notin P$, since $P \cap A=$ $p$, by (3). Further, $d \notin P B_{a}$; for $B_{a}$ is integrally dependent on $D$, hence height $P B_{a} \cap D=1$, since $D$ is integrally closed, and so $p D=$ $P B_{a} \cap D$ and $d \notin p D$. Therefore, $B_{P}=\left(B_{a d}\right)_{P_{a d}}$ and $D_{d}[b] \subseteq B_{a d} \subseteq D_{d}^{\prime}=$ $D_{d}[b]$. Hence, since $p D_{d}^{\prime}$ is unramified over $A, P B_{P}$ is unramified over $A$, and so $p B$ is unramified over $A$.

REMARKS 4.5. (1) In (2) of (4.4), the condition: "Height $P \cap$ $A \leqq 1$, for all height one prime ideals $P$ in $B$ "; is equivalent to: "Height $Q B>1$, for all prime ideals $Q$ in $A$ such that height $Q>1$. Also, the condition: "Only finitely many of the ideals $p B$ have imbedded prime divisors", is equivalent to: "If $P$ is an imbedded prime divisor of $p B$, then $P \cap A=p$ ", by (4.2)(1) and (2) and (2.9)(1).

(2) A regular domain satisfies the conditions on $A$ in (4.4).

(3) The proof of (4.4) shows that $(1) \Rightarrow(2) \Longrightarrow(3)$ for an arbitrary Noetherian domain which contains $A$, and $(3) \Rightarrow(1)$ with $\mathscr{C}$ replacing $B$ in the statements, where either $\mathscr{C}=B^{\prime}$ or $\mathscr{C}$ is a Noetherian ring such that $B \subseteq \mathscr{C} \subseteq B^{\prime}$. (That $\mathscr{I}(\mathscr{C})$ is finite follows from: $a \mathscr{C}$ and $d \mathscr{C}_{a}$ have only finitely many imbedded prime divisors and $\mathscr{C}_{a d}=D_{d}^{\prime}$.)

Corollary 4.6. (Cf. [4, Theorem 5].) If $B$ is a finite separably generated extension domain of a Dedekind domain A, then (1)-(3) of (4.4) hold.

Proof. $A$ is a locally factorial Noetherian domain, and (3) in (4.4) is satisfied.

Corollary 4.7. Let $A$ and $B$ be as in (4.4), and assume that $A$ is a regular local domain of altitude two. If $M B=B$, where $M$ is the maximal ideal in A, then (1)-(3) of (4.4) hold.

Proof. The proof is the same as the proof of (4.6).

Corollary 4.8. Let $A$ and $B$ be as in (4.4), and assume that $B$ is a flat A-algebra. Then (1)-(3) of (4.4) hold.

Proof. (3) in (4.4) is satisfied.

Corollary 4.9. Let $A$ and $B$ be as in (4.4), and assume (1)-(3) 
hold. Then $B_{P}$ is a regular local ring, for all except finitely many height one prime ideals $P$ in $B$.

Proof. Let $P$ be a height one prime ideal in $B$. If $P \cap A \neq(0)$, then height $P \cap A=1$ (4.4)(3), and only finitely many such $P$ fail to satisfy $B_{P}$ is a regular local ring (4.4)(1). If $P \cap A=(0)$, then let $S=A \sim(0)$, let $F=A_{S}$, and let $C=B_{S}$. Then $D_{S}$ is a finite separably generated extension domain of the field $F$, hence it is well known [5, (36.6)] that the integral closure $C^{\prime}$ of $C$ is a finite $C$-algebra. Therefore, only finitely many height one prime ideals $Q$ in $C$ fail to satisfy $C_{Q}$ is a regular local ring (namely those $Q$ which contain $C: C^{\prime}$ ). Since $P C$ is a height one prime ideal, for all such $P$ (height $P=1$ and $P \cap A=(0))$, the conclusion follows.

CoRollary 4.10. Let $A$ and $B$ be as in (4.4), let $\mathscr{C}$ be a ring such that either $\mathscr{C}=B^{\prime}$, the integral closure of $B$, or $\mathscr{C}$ is Noetherian and $B \subseteq \mathscr{C} \subseteq B^{\prime}$, and let $\mathscr{H}=\{P \in$ Spec $\mathscr{C}$; height $P=1 \geqq$ height $P \cap A\}$. Then $\mathscr{C}_{P}$ is a regular local ring, for all except finitely many $P \in \mathscr{H}$.

Proof. Only finitely many $P \in \mathscr{H}$ such that $P \cap A=(0)$ fail to satisfy $\mathscr{C}_{P}$ is a regular local ring, as in the proof of (4.9). Also, by the proof that $(3) \Rightarrow(1)$ in (4.4), for all $P \in \mathscr{H}$ such that $P \cap A \neq(0)$ and $a \notin P$ and $d \notin P \mathscr{C}_{a}, \quad \mathscr{C}_{P}$ is a regular local ring. The conclusion follows from this.

\section{REFERENCES}

1. D. Ferrand and M. Raynaud, Fibres formelles d'un anneau local Noethérian, Ann. Sci. Ecole Norm. Sup., 3 (1970), 295-311.

2. A. Grothendieck, Elements de Géométrie Algebrique, IV (Première Partie), Paris: Institut des Hautes Etudes Scientifiques, 1964.

3. - Elements de Géométrie Algebrique, IV (Seconde Partie), Paris: Institut des Hautes Etudes Scientifiques, 1965.

4. M. Nagata, A general theory of algebraic geometry over Dedekind domains, II, Amer. J. Math., 80 (1958), 382-420.

5. — Local Rings, New York, Interscience, 1962.

6. D. G. Northcott and D. Rees, Principal systems, Quart. J. Math. Oxford Ser., 8 (1957), 119-127.

7. L. J. Ratliff, Jr., On quasi-unmixed local domains, the altitude formula, and the chain condition for prime ideals (II), Amer. J. Math., 92 (1970), 99-144.

8. - On prime divisors of the integral closure of a principal ideal, J. Reine Angew. Math., forthcoming.

9. D. Rees, $A$ note on form rings and ideals, Mathematika, 4 (1957), 51-60.

Received July 5, 1972. Work on this paper was supported in part by the National Science Foundation Grant NSF-28939. 


\section{PACIFIC JOURNAL OF MATHEMATICS}

\section{EDITORS}

RICHARD ARENS (Managing Editor) University of California

Los Angeles, California 90024

R. A. Beaumon'T

University of Washington Seattle, Washington 98105
J. Dugundj1*

Department of Mathematics University of Southern California Los Angeles, California 90007

D. Gilbarg and J. Milgram Stanford University Stanford, California 94305

\section{ASSOCIATE EDITORS}

E. F. BECKENBACH

B. H. NeUMANN

F. WOLF

K. YOSHIDA

\section{SUPPORTING INSTITUTIONS}

UNIVERSITY OF BRITISH COLUMBIA CALIFORNIA INSTITUTE OF TECHNOLOGY UNIVERSITY OF CALIFORNIA MONTANA STATE UNIVERSITY UNIVERSITY OF NEVADA NEW MEXICO STATE UNIVERSITY OREGON STATE UNIVERSITY UNIVERSITY OF OREGON OSAKA UNIVERSITY

\section{UNIVERSITY OF SOUTHERN CALIFORNIA STANFORD UNIVERSITY UNIVERSITY OF TOKYO UNIVERSITY OF UTAH WASHINGTON STATE UNIVERSITY UNIVERSITY OF WASHINGTON AMERICAN MATHEMATICAL SOCIETY NAVAL WEAPONS CENTER}

* C. R. DePrima California Institute of Technology, Pasadena, CA 91109, will replace J. Dugundji until August 1974. 


\section{Pacific Journal of Mathematics}

\section{Vol. 49, No. $1 \quad$ May, 1973}

A. Bigard, Free lattice-ordered modules ...........................

Richard Bolstein and Warren R. Wogen, Subnormal operators in strictly cyclic

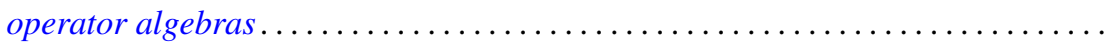

Herbert Busemann and Donald E. Glassco, II, Irreducible sums of simple

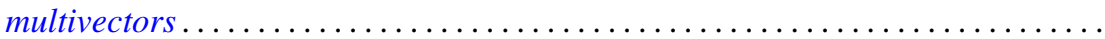

W. Wistar (William) Comfort and Victor Harold Saks, Countably compact groups

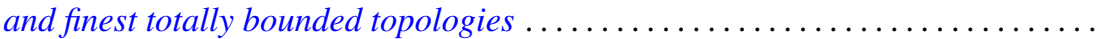

Mary Rodriguez Embry, Maximal invariant subspaces of strictly cyclic operator

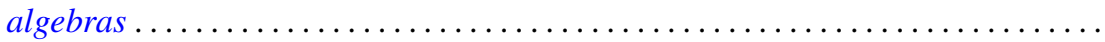

Ralph S. Freese and James Bryant Nation, Congruence lattices of semilattices......

Ervin Fried and George Grätzer, A nonassociative extension of the class of

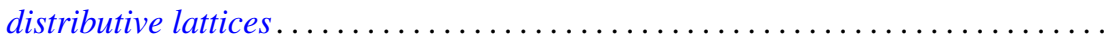

John R. Giles and Donald Otto Koehler, On numerical ranges of elements of locally

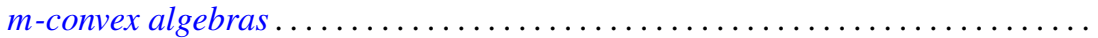

David A. Hill, On dominant and codominant dimension of $\mathrm{QF}-3$ rings ........ John Sollion Hsia and Robert Paul Johnson, Round and Pfister forms over $R(t) \ldots$ I. Martin (Irving) Isaacs, Equally partitioned groups . . . . . . . . . . . . . .

Athanassios G. Kartsatos and Edward Barry Saff, Hyperpolynomial approximation of solutions of nonlinear integro-differential equations.

Shin'ichi Kinoshita, On elementary ideals of $\theta$-curves in the 3-sphere and 2-links in the 4-sphere

Ronald Brian Kirk, Convergence of Baire measures

R. J. Knill, The Seifert and Van Kampen theorem via regular covering spaces ..

Amos A. Kovacs, Homomorphisms of matrix rings into matrix rings ..

Young K. Kwon, HD-minimal but no $H D$-minimal ..........

Makoto Maejima, On the renewal function when some of the mean renewal lifetimes are infinite

Juan José Martínez, Cohomological dimension of discrete modules over profinite groups.

W. K. Nicholson, Semiperfect rings with abelian group of units

Louis Jackson Ratliff, Jr., Three theorems on imbedded prime divisors of principal ideals.

Billy E. Rhoades and Albert Wilansky, Some commutants in $B(c)$ which are almost matrices

John Philip Riley Jr., Cross-sections of decompositions . . .

Keith Duncan Stroyan, A characterization of the Mackey uniformity $m\left(L^{\infty}, L^{1}\right)$ for

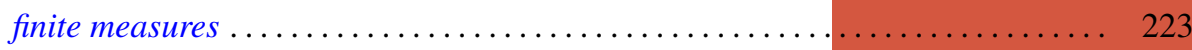

Edward G. Thurber, The Scholz-Brauer problem on addition chains . . . . . . . . . 229

Joze Vrabec, Submanifolds of acyclic 3-manifolds ............

Philip William Walker, Adjoint boundary value problems for compactified singular

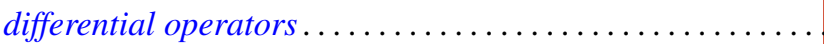

